\title{
An Optimal Penetration of Distributed Generation Decision Method for Distribu- tion System
}

\author{
Xiangyu Kong ${ }^{1}$, Shuai Zhao ${ }^{1}$, Jidong Wang ${ }^{1}$, Kai Wang ${ }^{2}$, Li Gang ${ }^{2}$ \\ 1. Key Laboratory of Smart Grid of Ministry of Education, Tianjin University, China \\ 2. Electric Power Research Institute of Guangdong Power Grid Corporation, China
}

\begin{abstract}
:
The impact of distributed generation on a local sub-transmission system or a local distribution system was studied in the paper. DGs operating models for dynamic stability analysis were obtain, and an optimal penetration of DGs decision method was proposed to maximize the penetration level of DG on the system. A modified IEEE 39-Bus test system with DGs is used to prove the validity of the proposed method.
\end{abstract}

Keywords: distributed generation; stability analysis; power system; assessment

\section{Introduction}

Distributed generation can provides customers with better quality, more reliable energy supplies and a cleaner environment. However, it is necessary to known the impact of high permeability DGs on power system and their reliability assessment. Interconnection of DG can create operation and control problems [1], especially a large penetration level of photovoltaic (PV) or wind turbine generation, which cannot control power output, causing voltage fluctuation.

In the future, the penetration level of DGs will be increased in insignificant number. With a power system having a large size of aggregate DGs, system instability is difference settle down to a new equilibrium point within an acceptable time frame. Both periodic oscillations and small fluctuations in the voltage and frequency are not desirable and cannot be accepted in the post disturbance state [2]. The impact study of steady-state and dynamic stability analyses with uncontrollable DGs power output have to be performed in references [3].

Though most dynamic stability analyses of a large transmission system only concern three-phase fault, large load switching and tripping-off generator may affect to dynamic performance of the local sub-transmission and distribution system [4-5]. The dynamic stability of the system normally concentrates on a rotor angle stability for both synchronous and induction generation. Nonetheless, during a disturbance occurring in the system with high permeability DGs, power outputs of machines, system frequency, and system voltage are changed. Some instabilities occurring in the system come from voltage instability due to lack of reactive power support. Moreover, various factors, such as generators parameters and controls, strength of the system, and protection scheme, are important to the stability of power system [6].

The purpose of this paper is to study the impact of DG on a local distribution system when experiencing large disturbances so that local utilities can find ways to limit the dynamic impact of DG to an acceptable level and an energy sensitivity 
approach for simultaneously processing a set of severe stability. The protection setting is also discussed to maximize the penetration level of DG on the system.

\section{DGs operating characteristics and models for dynamic stability analy- sis}

\subsection{DG characteristics for stability re- search}

As DG ranges in size from a kilowatt up to tens of megawatts and can be connected to the distribution or transmission system and can sell electricity to the utility or to its own customers. Some types of DGs also provide heat or steam for use at or near the site to maximize efficiency. DG technologies can be categorized into the following three groups: (1) Combined Heat and Power, such as CHP; (2) Renewable energy resources, such as solar electric, wind turbines, or small hydroelectric generator; (3) Reciprocating engine generators and small combustion turbines [4].

Since DGs have different operating characteristics and their impacts to the systems are different, based on the types of generators, in this paper, DGs be divided into synchronous generator, induction generator, and inverter-based generator. Synchronous and induction generator are typical for most of DG technologies except solar electric systems and fuel cells, which are inverter-based DG.

\subsection{Typical DG model for dynamic sta- bility analysis}

1) Synchronous machine model

A model for a synchronous machine can be described as

$$
\frac{2 H}{w b} \frac{d}{d t}\left(w_{r}\right)=P_{T}-P_{e}-D w_{r}
$$

Where $H$ is an inertia time constant for a synchronous machine; $w_{b}$ and $w_{r}$ are the rated speed and rotor speed for a synchronous machine, respectively; $P_{T}$ and $P_{e}$ describe the mechanical power input and electrical power, respectively, and $D$ show the damping coefficient.

In a simple model for a synchronous machine, $P_{T}$ and $P_{e}$ are assumed to be constant. And the simple synchronous machine model is

$$
\begin{aligned}
& w_{r}(s)=\frac{P_{T}(s)-P_{e}(s)}{D+T_{P}} \\
& T_{P}=s \frac{2 H}{w_{b}}
\end{aligned}
$$

And the simple synchronous machine block diagram is shown in Fig. 1.

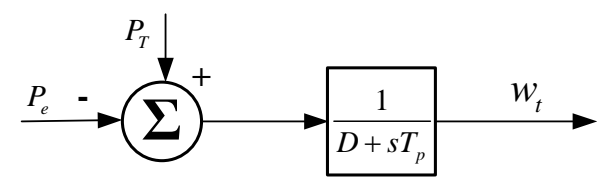

Fig. 1 Simple synchronous machine model

2) Induction generator model

Induction machine models of varying complexity have been used. A standard fifth order model expressed in the $d-q$ coordinate system has been used in reference [6]. The general power equation for the induction generator is as follows:

$$
\Delta P=P_{e}-P_{M}
$$

where

$$
\begin{aligned}
& U_{s}=R_{S} i_{S}+\frac{d}{d t}\left[L_{s} i_{s}+L_{m} i_{r}\right] \\
& L_{s}=L_{m}+L_{s \lambda} \\
& L_{r}=L_{m}+L_{r \lambda} \\
& R_{m}=R_{r}(1-s) / s
\end{aligned}
$$

Where: $U_{s}$ is stator voltage, $i_{s}$ and $i_{r} d e$ scribes stator current and rotor current, respectively; $R_{S}$ stator winding resistance and $R_{r}$ rotor winding resistance referred 
to the stator side; $L_{m}$ and $R_{m}$ describes generator magnetizing inductance and resistance, $L_{s \lambda}$ and $L_{r \lambda}$ are stator leakage inductance and rotor leakage inductance referred to the stator side, respectively; $R_{r}$ is the stator resistance for the induction machine.

The wind generation unit which connects to the grid is shown in Fig. 2.

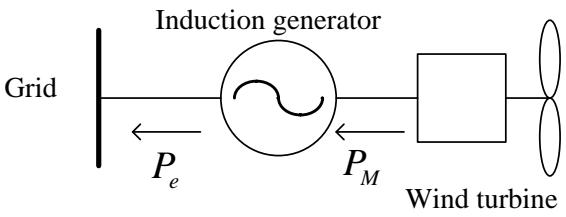

Fig.2 Induction generator models for wind turbine

\section{3) Diesel engine dynamic model}

The differences between the desired speed and the synchronous machine speed or engine speed produce the control signal, which causes a change in the torque of the prime mover. The diesel engine generates the torque and the mechanical power would be given in the following equation as

$$
P_{m}=w \times T_{e}
$$

where $P_{m}$ is mechanical power, $w_{r}$ represent Rated Speed, and $T_{e}$ is the engine torque.

Then measured synchronous machine speed is fed back to compare with the reference signal to control the governor. The diesel engine model is shown as Fig. 3.

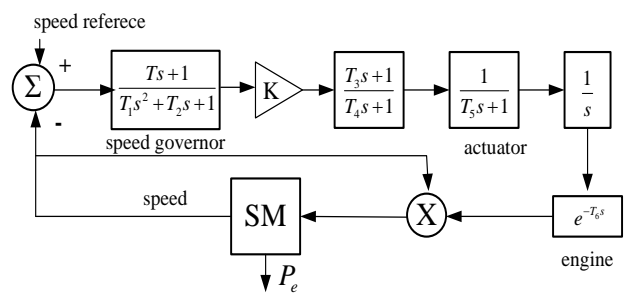

\section{Impact of DGs on the Distribution Protection and Stability}

\subsection{Characters of GDs with system congestion}

With the penetration level of DGs increases, if there is a phase fault at a branch circuit of a distribution system, DGs will contribute fault current. When a system congestion happened, such as a three-phase shorten, fault current from the main grid through distribution substation is added up by the fault current from DGs, therefore, the total fault current is increased. Because of the infeed effect, the fault current contributed from the main grid is decreased.

In addition, with a higher fault current, a fuse is most likely operated before a fast operation of a recloser. The method to solve the problem due to reducing of reach is that the pick up current of overcurrrent relays should be decreased, but it should have a capability to classify between fault and load current. Although the fault is located on between the substation and DGs, this scheme can also be employed except no recloser curves.

\subsection{Stability performance with high permeability DGs}

While steady-state operation the system is balanced between the system power output and consumed. However, but a power system is never in the steady state because the loads and generations are continuously changing. Dynamics of fault-on system is represented by the following differential-algebraic equations.

$$
\left\{\begin{array}{rl}
\dot{\mathrm{x}}(t) & =\mathrm{f}_{1}(\mathrm{x}, \mathrm{y}, \mathrm{u}) \\
0 & =\mathrm{g}_{1}(\mathrm{x}, \mathrm{y}, \mathrm{u})
\end{array} \quad t_{0} \leq t \leq t_{c l}\right.
$$

where vectors $\boldsymbol{x}, \boldsymbol{y}$ and $\boldsymbol{u}$ stand for state, algebraic and control variables; $t_{0}$ and $t_{c l}$ are for fault starting and clearing times.

Fig 3 Diesel engine model 
For a distribution system with high permeability DGs output, assumed that control generator set $S_{g}$ for every $j \in S_{C}$ is designated, all generators involving in the rescheduling control can be represented by DGs and conventional generation set. An optimal penetration of DGs decision method may be proposed as model (7).

$$
\begin{array}{ll} 
& \min C\left(\mathrm{P}_{g}\right) \\
\text { s.t. } & \sum_{i \in S_{g}} P_{g i}=\sum_{i \in S_{g}} P_{g i}^{0} \\
& J_{j}\left(\mathrm{x}\left(t_{f}\right), \mathbf{P}_{j, g}\right)<0,
\end{array}
$$$$
\text { for all contingency } j, j \in S_{C}
$$

Where, notation $C(\cdot)$ represents a output function; constraint emphasizes total generation of machines in keeps balance.

The evaluation of stability problems requires studying the respond of power system during and after the disturbances. But a system with high permeability synchronous model DGs, the frequency deviation at the generator bus will be used to represent a rotor angle deviation since the behavior of both parameters are nearly the same including the duration of oscillation, but the amplitude of oscillation of a rotor angle deviation is a little higher. Besides, the frequency deviation is an important factor to examine the protection setting of DG during performing dynamic stability.

\section{Case study}

A modified IEEE 39-Bus Test System with Distributed Generation, as show in Fig.4, is used to modify the impact of DGs. This system is simple to interpret all results while at the same time it is complex enough show the robustness and ability of the algorithm to handle larger system. The DGs at bus 1, 3, 14, 16, 27 and 38 are wind generation, diesel engine and photovoltaic with the model in 2.2, which were incorporated to the test system and the power rating and terminal voltage of each DG is 5 to 7 MVA and 10 $\mathrm{kV}$, respectively, it includes.

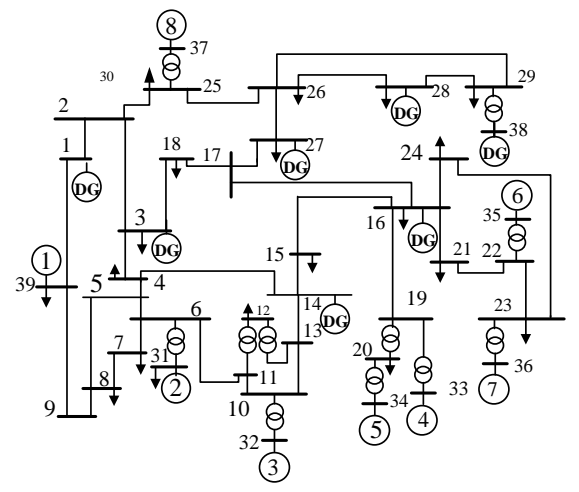

Fig.4 Modified IEEE 39-Bus Test System with distributed Generation

The severe cases are the disconnecting of larger capacity DG from the weakly connected power system. Table 1 shows results of some DGs disconnected from a system. Different disconnect at bus 3,26 and 27 out of the system by using under voltage relay. As expected, the larger the size of the tripping-off generator, the more voltage sag in distribution system. Voltages at the interconnection point decreases from 0.035 p.u. to 0.542 p.u. at bus 3 , and from 0.006 p.u. to 0.126 p.u. at bus 15 .

\begin{tabular}{|c|c|c|c|}
\hline \multirow[b]{2}{*}{$\begin{array}{l}\text { shutdown } \\
\text { DG bus }\end{array}$} & \multicolumn{3}{|c|}{ System performance after DG shutdown } \\
\hline & $\begin{array}{c}\text { Votage at } \\
\text { bus3 (p.u.) } \\
\text { Before/after }\end{array}$ & $\begin{array}{c}\text { Votage at } \\
\text { bus15 (p.u.) } \\
\text { Before/after }\end{array}$ & $\begin{array}{l}\text { transient } \\
\text { stability }\end{array}$ \\
\hline Bus 3 & $1.028 / 0.993$ & $1.012 / 1.006$ & stability \\
\hline Bus 27 & $1.028 / 0.911$ & $1.012 / 1.097$ & stability \\
\hline Bus $3+27$ & $1.028 / 0.803$ & $1.012 / 0.921$ & stability \\
\hline $\begin{array}{c}\text { Bus } \\
3+27+38\end{array}$ & $1.028 / 0.586$ & $1.012 / 0.886$ & instability \\
\hline
\end{tabular}

Table 1 Results with some DGs disconnected from system

As the stability is unstable due to voltage instability after disconnected DGs 
at bus $3+27+38$ for a congestion case at bus 21-22 since only one generator in weakly-connected area without any control devices to maintain the machine in stability as the SG does. The rotor angles of all Synchronous DGs and conventional generators are shown in Fig 5.

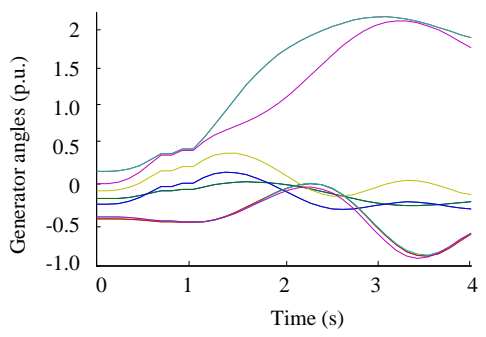

Fig.5 Swing angles of Synchronous DGs

Fig 6 shows the optimal real power and location of each DGs in order to maximize system stability. The real power remained constant indicating the algorithm reached on optimal solution. The main DG penetrations occurred at $10 \mathrm{kV}$ buses and DG values are shown in per unit on a 5 to $7 \mathrm{MW}$ base. The capacities of the DGs were $0.97,0.86,0.89,0.92$, $0.81,0.78$ p.u., which were connected at buses1, 3, 14, 16, 27 and 38 with 4.85 MW, 4.17 MW, 5.6 MW, 5.04 MW, 5.04 MW, 5.25 MW and 4.16 MW, respectively. The algorithm that determined the optimal DG location and capacity is easily and practical.

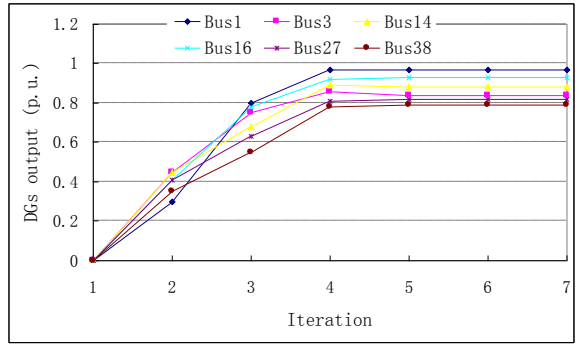

Fig. 6 DGs output with iteration generation

\section{Conclusion}

An optimal penetration of DGs decision method was proposed to maximize the penetration level of DG on the system in the paper. However, the impact of DG on the local systems depends on several parameters, such as type, size, location, operation mode of DG, load condition, and the strength of the interconnecting point. Some other impact of DG on both the steady-state and dynamic behaviors of power system should be investigated in the future.

\section{Acknowledgments}

This work supported by National High Technology Research and Development Program 863 of China (2011AA05A115).

\section{Reference}

[1] D. Gan, Chattopadhyay D, Xiaochuan Luo. "An improved method for optimal operation under stability constraints,". In Proc. IEEE Power Eng. Soc. Transmiss. Distrib. Conf., Dallas, US, 2003, vol. 2, pp. 683-688

[2] Vladislav A, Hans K, Arne H N, et al. "Modeling and transient stability of large wind farms", 2nd International Workshop on Transmission Networks for Offshore Wind Farms, 2001, Royal Institute of Technology, Stockholm, Sweden.

[3] Xiangyu Kong, Dazhong Fang, Chung T S. "Economy and reliability evaluation of generating including wind energy systems". 3rd International Conference on Deregulation and Restructuring and Power Technologies, Nanjing, China, Apr, 2008, PP: $2653-$ 2657.

[4] Chompoo I, Chai Lee, Wei-Jen. "System Impact Study for the Interconnection of Wind generation and utility system". IEEE Transactions on Industry Applications, 2005, 41(1): 163 168. 
[5] Asber D L, Serge H M. "Transient Behavior of a Distribution Network Incorporating Decentralized Generation". International Journal of Electrical Power and Energy Systems, 2005, 27(3): 195-203.
[6] Feng Xingtian, Wei Tongzhen, Kong Lingzhi. "Influence of High Permeability Distributed Generation on Voltage Quality of Distribution Network". Water Resources and Power, 2010, 28(9): 154-157. 